\title{
Soluble fibrinogen-like protein 2 levels are decreased in patients with ischemic heart failure and associated with cardiac function
}

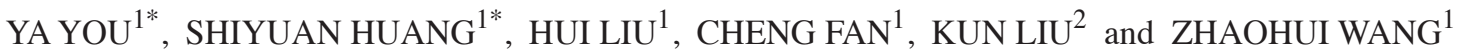 \\ Departments of ${ }^{1}$ Geriatrics and ${ }^{2}$ Cardiology, Union Hospital, Tongji Medical College, \\ Huazhong University of Science and Technology, Wuhan, Hubei 430022, P.R. China
}

Received December 18, 2020; Accepted April 26, 2021

DOI: $10.3892 / \mathrm{mmr} .2021 .12198$

\begin{abstract}
Soluble fibrinogen-like protein 2 (sFGL2), as a novel effector of regulatory $\mathrm{T}$ cells (Tregs), exhibits immune regulatory activity in several inflammatory diseases. Immune activation and persistent inflammation participate in the progression of ischemic heart failure (IHF). The present study aimed to determine serum sFGL2 levels in patients with IHF and explore the relationship between sFGL2 levels and cardiac function. A total of 104 patients with IHF and 32 healthy controls were enrolled. Patients with IHF were further split into subgroups according to the New York Heart Association functional classification or left ventricular ejection fraction (LVEF). Serum sFGL2 levels and peripheral Tregs frequencies were analyzed by ELISA and flow cytometry, respectively. The suppressive function of Tregs was measured by proliferation and functional suppression assays. Serum levels of sFGL2 and circulating Tregs frequencies were significantly decreased in patients with IHF compared with healthy controls. In patients with IHF, sFGL2 levels and Tregs frequencies were decreased with the deterioration of cardiac function. Tregs from patients with IHF exhibited compromised ability to suppress $\mathrm{CD} 4^{+} \mathrm{CD} 25^{-} \mathrm{T}$ cells proliferation and inflammatory cytokines secretion. Specifically, sFGL2 levels and Tregs frequencies positively correlated with LVEF, whereas negatively correlated with left ventricular end-diastolic dimension and N-terminal pro-brain natriuretic peptide. sFGL2 levels were positively correlated with Tregs frequencies. In conclusion, the reduction of serum sFGL2 levels are associated with the progression of IHF and sFGL2 could be used as a potential indicator for predicting disease severity.
\end{abstract}

Correspondence to: Dr Zhaohui Wang, Department of Geriatrics, Union Hospital, Tongji Medical College, Huazhong University of Science and Technology, 1277 Jiefang Avenue, Wuhan, Hubei 430022, P.R. China

E-mail: zhaohuiwang@hust.edu.cn

${ }^{*}$ Contributed equally

Key words: soluble fibrinogen-like protein 2, regulatory $\mathrm{T}$ cells, ischemic heart failure, adverse ventricular remodeling

\section{Introduction}

Advanced treatments have effectively reduced the deaths from myocardial infarction (MI), however the morbidity and mortality of post-infarction ischemic heart failure (IHF) are increasing and becoming a major threat to human health worldwide (1-3). Adverse ventricular remodeling (AVR) following MI is the pathological basis of IHF and is largely ascribed to inflammatory responses (4-6). Various immune cells, including $\mathrm{T}$ helper lymphocytes, regulatory $\mathrm{T}$ cells (Tregs), monocytes and macrophages, participate in the complicated process $(7,8)$. Notably, Tregs, which are regarded to be a subset of anti-inflammatory lymphocytes, act as a key player in immune suppression and modulate the immune balance (9-11).

Recently, Tregs have shown to play an important role in cardiovascular diseases such as dilated cardiomyopathy (12), hypertension (13), atherosclerosis (14), acute coronary syndrome (ACS) (15) and chronic heart failure (CHF) $(9,16)$. Clinical studies report that a reduced number and impaired function of Tregs are present in CHF $(9,10,17)$. The underlying mechanism may be attributed to impaired thymic output of nascent Tregs and increased cell apoptosis in periphery (10). Following ischemic injury, high sensitivity to FasL-induced apoptosis or IL-2 deprivation may also contribute to the defect of Tregs in patients with CHF (10). Tregs can improve cardiac repair and attenuate AVR following MI by inhibiting inflammation and cardiomyocytes damage through cell-to-cell contact and inhibitory cytokines production, including IL-10 and TGF- $\beta$ (17). Additionally, a novel effector molecule of Tregs, sFGL2, has attracted attention as an immunoregulatory factor.

sFGL2 belongs to the fibrinogen-related proteins superfamily, which is mainly secreted by Tregs $(18,19)$. It exhibits immune regulatory activity in autoimmune glomerulonephritis (20), renal allograft acute rejection (21) and viral hepatitis (22). Recent evidence has indicated that sFGL2 is able to suppress the immune response by inhibiting dendritic cell maturation and $\mathrm{T}$ cell proliferation (23). Downregulation of sFGL2 inhibits differentiation and activity of $\mathrm{CD}^{+}{ }^{+} \mathrm{CD} 25^{+} \mathrm{Foxp}^{+}$Tregs (23). Based on the observation that sFGL2 may be involved in the modulation of inflammatory responses, it was hypothesized that sFGL2 may play a role in IHF. 
Our previous study observed that serum levels of sFGL2 were markedly reduced in patients with ACS, in accordance with significantly decreased frequencies of Tregs (24). Long-term coronary ischemia and inflammatory activation can inevitably give rise to myocardial damage in ACS patients. Since we had demonstrated an altered status of sFGL2 in vascular events, it was hypothesized that sFGL2 would be implicated in the development of cardiac dilatation and dysfunction. Thus, the present study aimed to measure the serum sFGL2 levels to explore their possible changes in patients with IHF and to further assess the association between sFGL2 levels and cardiac function.

\section{Materials and methods}

Subjects. To match the sample size of each group, 136 subjects were enrolled from the Union Hospital of Huazhong University of Science and Technology (Wuhan, China) between September 2017 and December 2018 in the present prospective study (Table I). These subjects were divided into a healthy control group $(n=32)$ and a IHF group $(n=104)$. In order to investigate the relationship between serum sFGL2 levels and cardiac function, the 104 patients with IHF were classified into subgroups according to the New York Heart Association (NYHA) functional classification (25) or left ventricular ejection fraction (LVEF) (25). Based on NYHA classification, the patient subgroups were NYHA I-II $(\mathrm{n}=57)$ and NYHA III-IV $(\mathrm{n}=47)$. Additionally, according to LVEF, the patients with IHF were subdivided into a heart failure with preserved ejection fraction (HFpEF) group (LVEF $\geq 50 \%, n=37$ ), heart failure with mid-range ejection fraction (HFmrEF) group (LVEF 40-49\%, n=32) and the heart failure with reduced ejection fraction (HFrEF) group (LVEF $<40 \%, \mathrm{n}=35$ ). The study was approved by the Ethics Committee of Tongji Medical College, Huazhong University of Science and Technology (approval. no. IORG0003571) and written informed consent was obtained from each participant. The present study conformed to the Declaration of Helsinki principles.

Inclusion criteria. CHF was diagnosed by clinical history, physical examination, electrocardiography, chest X-ray, echocardiography and $\mathrm{N}$-terminal pro-brain natriuretic peptide (NT-proBNP). Patients with CHF were eligible for IHF if they had a history of MI or prior revascularization, or $\geq 50 \%$ stenosis in two or more major epicardial coronary artery. For comparison, 32 healthy volunteers from a routine health examination were enrolled as normal controls, who had no symptoms or signs of IHF.

Exclusion criteria. Patients with the following diseases were excluded from the study: Valvular heart disease, rheumatic heart disease, idiopathic dilated cardiomyopathy, hypertrophic cardiomyopathy, serious infection, virus hepatitis, autoimmunity disease, renal failure and malignant tumors.

Blood samples. Peripheral blood samples were obtained from all patients in a fasting state with clean venipuncture of an antecubital vein. The first $4 \mathrm{ml}$ were collected into a heparinized tube and was used for flow cytometric analysis, while the subsequent $2 \mathrm{ml}$ were collected into a sterile tube without the anticoagulation for ELISA. Peripheral blood mononuclear cells (PBMCs) were isolated by Ficoll density gradient centrifugation $\left(400 \mathrm{x} \mathrm{g}, 4^{\circ} \mathrm{C}, 25 \mathrm{~min}\right)$ and then used for flow cytometric analysis. Serum was collected following centrifugation and frozen at $-80^{\circ} \mathrm{C}$ until analysis.

Flow cytometric analysis of Tregs. PBMCs were washed two times and suspended with PBS at a density of $1 \times 10^{7}$ cell $/ \mathrm{ml}$. $100 \mu \mathrm{l}$ of the cell suspension was transferred to $1 \mathrm{ml}$ Eppendorf tube and then the cells were incubated with anti-human CD4-FITC (1:50; cat. no. 11-0049-42; eBioscience; Thermo Fisher Scientific, Inc.) and anti-human CD25-PE (1:50; cat.no. 12-0259-80; eBioscience; Thermo Fisher Scientific,Inc.) at $4{ }^{\circ} \mathrm{C}$ for $30 \mathrm{~min}$. Following the surface staining, the cells were washed once and resuspended in $1 \mathrm{X}$ fixation/permeabilization buffer (500 $\mu \mathrm{l})$ according to the manufacturer's instructions (eBioscience; Thermo Fisher Scientific, Inc.), followed by two washes in 1X permeabilization buffer for Foxp3 intracellular staining. The cells were then stained with anti-human Foxp3-APC (1:50; cat. no. 17-4776-42; eBioscience; Thermo Fisher Scientific, Inc.) at $4^{\circ} \mathrm{C}$ for $30 \mathrm{~min}$. Isotype controls were given to insure correct compensation and antibody specificity. All of the antibodies used for flow cytometry were from eBioscience (Thermo Fisher Scientific, Inc.). Stained cells were analyzed on a fluorescence-activated cell sorting (FACS) flow cytometer (FACS Aria; BD Biosciences). All data were analyzed using FlowJo V7.6.1 (FlowJo LLC).

Proliferation and functional suppression assays. PBMCs from controls $(\mathrm{n}=10)$ and patients with IHF $(\mathrm{n}=10)$ were stained with anti-human CD4-FITC (1:50; cat. no. 11-0049-42; eBioscience; Thermo Fisher Scientific, Inc.) and anti-human CD25-PE (1:50; cat. no. 12-0259-80; eBioscience; Thermo Fisher Scientific, Inc.) at $4^{\circ} \mathrm{C}$ for $30 \mathrm{~min}$. Following surface staining, $\mathrm{CD}^{+}{ }^{+} \mathrm{CD} 25^{-} \mathrm{T}$ cells (responder $\mathrm{T}$ cells; Tresp) and $\mathrm{CD} 4{ }^{+} \mathrm{CD} 25^{+}$Tregs were obtained by FACS sorting using a FACS Aria (BD Biosciences). $\mathrm{CD} 4^{+} \mathrm{CD} 25^{-} \mathrm{T}$ cells were suspended with PBS at a density of $2 \times 10^{6} \mathrm{cell} / \mathrm{ml}$ and labelled with $2 \mu \mathrm{M}$ carboxyfluorescein succinimidyl ester (CFSE; Thermo Fisher Scientific, Inc.) at $37^{\circ} \mathrm{C}$ in $5 \% \mathrm{CO}_{2}$ for $10 \mathrm{~min}$ while rotating once at $5 \mathrm{~min}$. Then CFSE-labelled Tresp cells (1x $10^{5} /$ well) from controls or IHF group were cultured alone or co-cultured with $\mathrm{CD} 4{ }^{+} \mathrm{CD} 25^{+}$Tregs from controls or IHF group at different ratio of 1:1, 2:1, 4:1 and 8:1 with stimulation of plate-bound anti-CD3 $(10 \mu \mathrm{g} / \mathrm{ml}$; eBioscience; Thermo Fisher Scientific, Inc.) and soluble anti-CD28 $(5 \mu \mathrm{g} / \mathrm{ml}$; eBioscience; Thermo Fisher Scientific, Inc.). All cells were incubated in complete RPMI 1640 supplemented with 10\% fetal bovine serum (Invitrogen; Thermo Fisher Scientific, Inc.) with a final volume of $200 \mu 1 /$ well in 96-well plates (BD Biosciences) at $37^{\circ} \mathrm{C}$ and $5 \% \mathrm{CO}_{2}$ for 4 days. Proliferation of Tresp cells were analyzed by flow cytometry based on CFSE dilution of gated $\mathrm{CD}^{+} \mathrm{T}$ cells. The percentages of $\mathrm{CFSE}^{\text {low }}$ cells cultured alone or co-cultured with Tregs in each group were analyzed, and then the reduced proportions of these percentages in co-culture system were calculated as follows: (A-B)/A, where $\mathrm{A}$ is the percentage of CFSE ${ }^{\text {low }}$ Tresp cells cultured alone and $\mathrm{B}$ is the percentage of $\mathrm{CFSE}^{\text {low }}$ Tresp cells co-cultured with Tregs. 
Table I. Baseline characteristics of healthy controls and patients with IHF.

\begin{tabular}{|c|c|c|c|}
\hline Characteristics & Controls $(n=32)$ & IHF $(n=104)$ & P-value \\
\hline Age (years) & $65.0 \pm 9.5$ & $65.88 \pm 10.3$ & 0.666 \\
\hline Sex (male/female) & $23 / 9$ & $86 / 18$ & 0.180 \\
\hline \multicolumn{4}{|l|}{ Echocardiography data } \\
\hline LVEF (\%) & $65.3 \pm 4.5$ & $45.8 \pm 13.3$ & $<0.0001$ \\
\hline LVEDD (cm) & $4.5 \pm 0.4$ & $5.8 \pm 0.5$ & $<0.0001$ \\
\hline \multicolumn{4}{|l|}{ NYHA classification } \\
\hline I-II (n) & - & 57 & - \\
\hline III-IV (n) & - & 47 & - \\
\hline NT-proBNP (pg/ml) & - & $2,875.27 \pm 1,263.30$ & - \\
\hline \multicolumn{4}{|l|}{ Risk factors } \\
\hline Hypertension, n (\%) & $17(53.1)$ & $70(67.3)$ & 0.144 \\
\hline Diabetes, n (\%) & $8(25.0)$ & $43(41.3)$ & 0.095 \\
\hline Hyperlipidemia, n (\%) & $18(56.3)$ & $76(73.1)$ & 0.072 \\
\hline Smoking, n (\%) & $9(28.1)$ & $46(44.2)$ & 0.105 \\
\hline
\end{tabular}

IHF, ischemic heart failure; LVEF, left ventricular ejection fraction; LVEDD, left ventricular end-diastolic dimension; NYHA, New York Heart Association; NT-proBNP, N-terminal pro-brain natriuretic peptide.

Detection of cytokines by ELISA. Serum levels of sFGL2 (cat. no. 436907; BioLegend, Inc.) and cell culture supernatant levels of IFN- $\gamma$ (cat. no. 430104; BioLegend, Inc.) and IL-17 (cat. no. 433914; BioLegend, Inc.) were measured using ELISA kits according to the manufacturer's instructions.

Statistical analysis. Continuous variables were expressed as mean \pm standard deviation in text and figures. Unpaired two-tailed Student's t-test was performed to detect two group differences and one-way analysis of variance (ANOVA) with Bonferroni's post-hoc test for multiple comparisons. Categorical data were expressed as frequencies and Chi-square test was performed for comparisons between groups. Pearson's correlation coefficients were used to establish associations between variables. Cut-off values for serum sFGL2 levels predicting IHF were analyzed by receiver operating characteristic (ROC) curve analysis. GraphPad Prism 5.0 (GraphPad Software, Inc.) and SPSS 19.0 (IBM Corp.) software were used for data analysis. $\mathrm{P}<0.05$ was considered to indicate a statistically significant difference.

\section{Results}

Basic clinical characteristics. The baseline characteristics of the 136 subjects are presented in Table I. Groups (Controls and IHF) were comparable with respect to age, gender and incidence of hypertension, diabetes, hyperlipidemia and smoking. Compared with controls, patients with IHF showed a lower LVEF and a larger left ventricular end-diastolic dimension (LVEDD). Table SI shows the demographic and clinical characteristics of controls and the NYHA I-II and NYHA III-IV groups. Basic clinical characteristics of controls and the HFpEF, HFmrEF and HFrEF groups are summarized in Table SII.
Serum sFGL2 levels and circulating Tregs frequencies are decreased in patients with IHF. Serum sFGL2 levels and peripheral Tregs frequencies were measured by ELISA and flow cytometry in patients with IHF, respectively. As shown in Fig. 1A, sFGL2 levels were noticeably reduced among patients with IHF $(11.90 \pm 6.48 \mathrm{ng} / \mathrm{ml})$ compared with controls $(18.27 \pm 5.69 \mathrm{ng} / \mathrm{ml} ; \mathrm{P}<0.0001)$. The frequencies of $\mathrm{CD} 4{ }^{+} \mathrm{CD} 25^{+} \mathrm{Foxp}^{+}$Tregs were significantly reduced in the IHF group $(1.99 \pm 0.81 \%)$ compared with controls $(4.99 \pm 0.67 \%$; $\mathrm{P}<0.0001$; Fig. 1B and C).

Serum sFGL2 levels and circulating Tregs frequencies are decreased with the deterioration of cardiac function in $I H F$. To investigate the relationship between sFGL2 levels, circulating Tregs frequencies and cardiac function in IHF, the patients were stratified into subgroups based on NYHA classification. As shown in Fig. 2A, sFGL2 levels were markedly reduced in the NYHA I-II group $(13.40 \pm 6.92 \mathrm{ng} / \mathrm{ml}$, $\mathrm{P}<0.001)$ and the NYHA III-IV group $(10.08 \pm 5.44 \mathrm{ng} / \mathrm{ml}$, $\mathrm{P}<0.001)$ compared with controls $(17.73 \pm 4.56 \mathrm{ng} / \mathrm{ml})$ and sFGL2 levels in the NYHA III-IV group were even lower than those in the NYHA I-II group $(\mathrm{P}<0.01)$. Similarly, the frequencies of $\mathrm{CD} 4{ }^{+} \mathrm{CD} 25^{+} \mathrm{Foxp} 3{ }^{+}$Tregs were markedly decreased in the NYHA I-II group $(2.48 \pm 0.72 \%, \mathrm{P}<0.001)$ and the NYHA III-IV group $(1.72 \pm 0.73 \%, \mathrm{P}<0.001)$ compared with controls $(4.85 \pm 0.51 \%$ ), while Tregs frequencies in the NYHA III-IV group were further decreased from those in the NYHA I-II group $(\mathrm{P}<0.001$; Fig. 2B and $\mathrm{C})$.

The above results implied that the reduction of sFGL2 and Tregs was intimately associated with cardiac function. To verify this, serum sFGL2 levels in patients with IHF with preserved, mid-range and reduced LVEF were analyzed. Fig. 3A shows that sFGL2 levels were prominently higher in the control group $(19.18 \pm 4.24 \mathrm{ng} / \mathrm{ml})$ than in the HFpEF group $(14.55 \pm 7.33 \mathrm{ng} / \mathrm{ml}$, $\mathrm{P}<0.05)$, the HFmrEF group $(11.43 \pm 5.41 \mathrm{ng} / \mathrm{ml}, \mathrm{P}<0.001)$ and the 
A

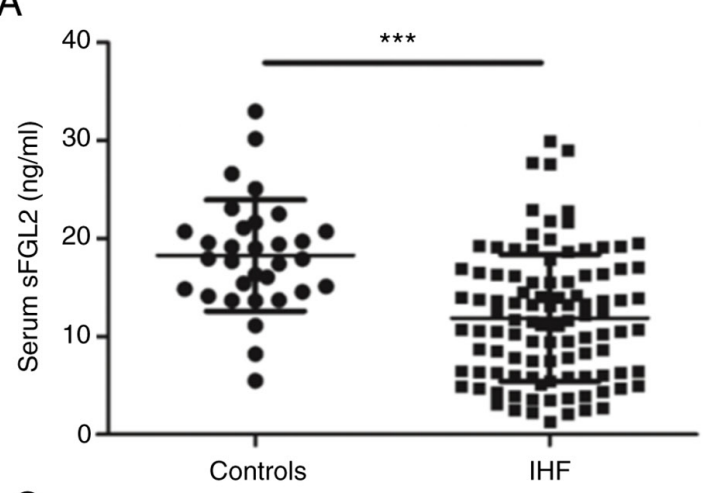

B

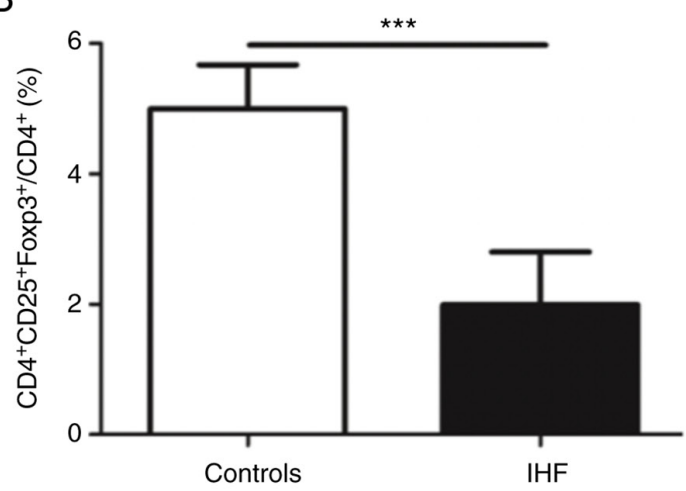

C
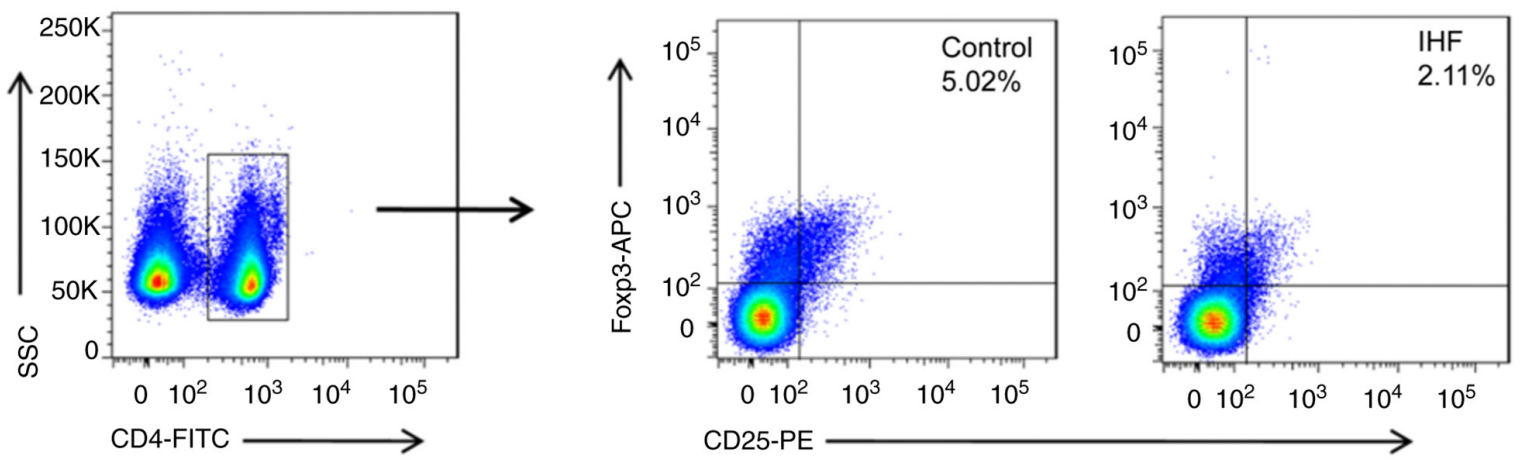

Figure 1. Serum sFGL2 levels and circulating Tregs frequencies are decreased in patients with IHF. (A) Serum sFGL2 levels were analyzed by ELISA. (B) The frequency of Tregs was compared in each group. (C) Representative FSC/SSC pseudo-color density image shows the gated CD4 $4^{+} \mathrm{T}$ cells and representative fluorescence-activated cell sorting plots of $\mathrm{CD} 4{ }^{+} \mathrm{CD} 25^{+} \mathrm{Foxp} 3^{+}$Tregs from a single patient in each group. ${ }^{* * *} \mathrm{P}<0.0001$. sFGL2, soluble fibrinogen-like protein 2 ; Tregs, regulatory T Cells; IHF, ischemic heart failure; FSC/SSC, forward scatter/side scatter.

A

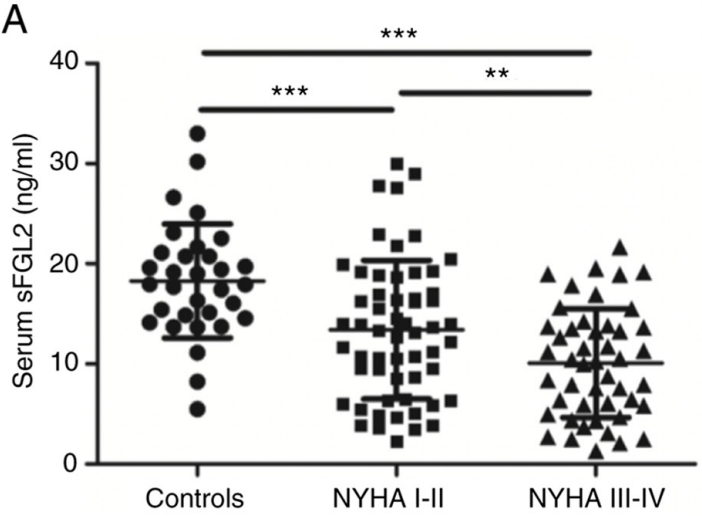

B

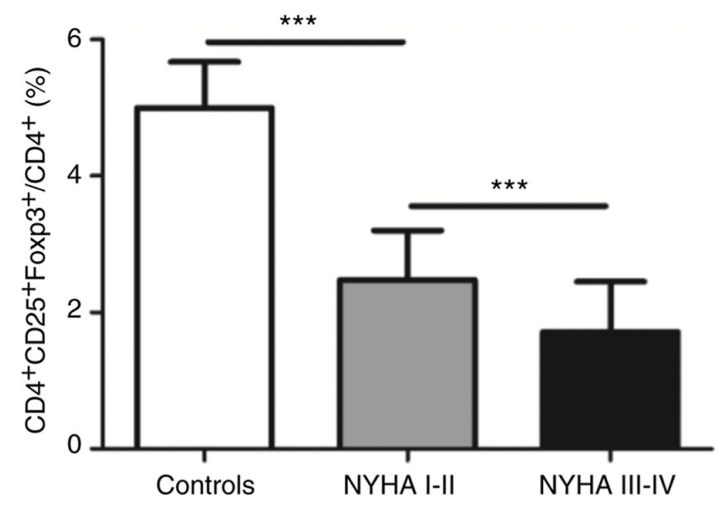

C
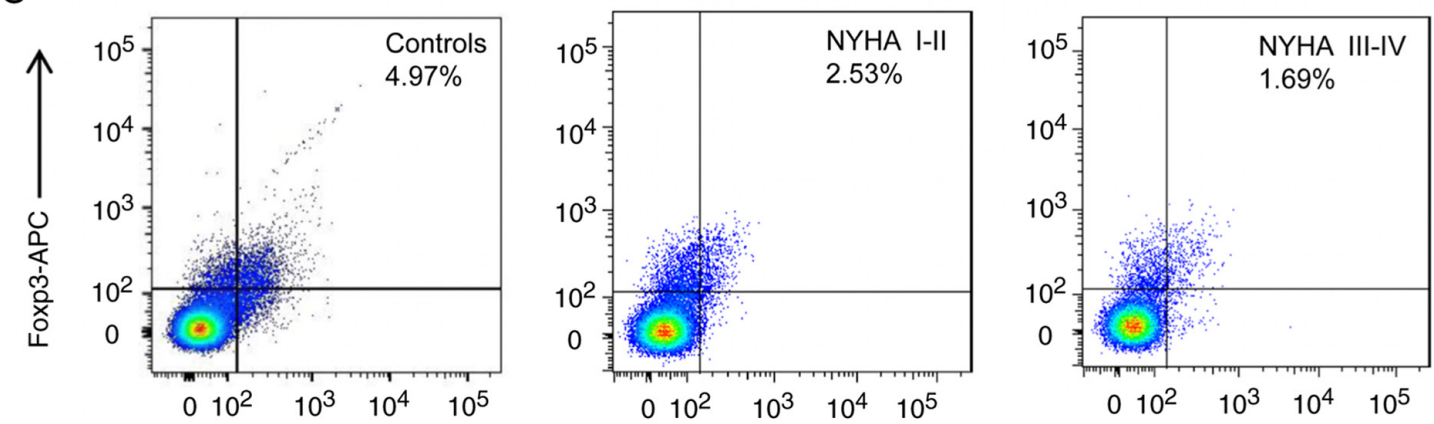

CD25-PE

Figure 2. Serum sFGL2 levels and circulating Tregs frequencies are decreased with the elevation of NYHA classification. (A) sFGL2 levels were analyzed by ELISA. (B) The proportion of Tregs was compared in each subgroup. (C) Representative fluorescence-activated cell sorting plots of CD $4^{+} \mathrm{CD} 25^{+} \mathrm{Foxp} 3^{+}$Tregs from a single person in each subgroup. ${ }^{* *} \mathrm{P}<0.01,{ }^{* * * *} \mathrm{P}<0.001$. sFGL2, soluble fibrinogen-like protein 2; Tregs, regulatory T Cells; NYHA, New York Heart Association. 



Figure 3. Serum sFGL2 levels and circulating Tregs frequencies are decreased with the reduction of LVEF. (A) sFGL2 levels were analyzed by ELISA. (B) The proportion of Tregs was compared in each subgroup. (C) Representative fluorescence-activated cell sorting plots of $\mathrm{CD}^{+} \mathrm{CD} 25^{+} \mathrm{Foxp} 3^{+}$Tregs from a single person in each subgroup. ${ }^{*} \mathrm{P}<0.05,{ }^{* *} \mathrm{P}<0.01,{ }^{* * *} \mathrm{P}<0.001$. sFGL2, soluble fibrinogen-like protein 2 ; Tregs, regulatory T Cells; LVEF, left ventricular ejection fraction; HFpEF, heart failure with preserved ejection fraction; HFmrEF, heart failure with mid-range ejection fraction; HFrEF, heart failure with reduced ejection fraction.

HFrEF group $(9.53 \pm 5.48 \mathrm{ng} / \mathrm{ml}, \mathrm{P}<0.001)$. Lower sFGL2 levels were observed in the HFmrEF group $(\mathrm{P}<0.05)$ and the HFrEF group $(\mathrm{P}<0.01)$ compared with the HFpEF group. However, no significant difference in sFGL2 levels were observed between the HFmrEF and HFrEF groups. Analogously, that the percentages of circulating $\mathrm{CD} 4^{+} \mathrm{CD} 25^{+} \mathrm{Foxp} 3^{+}$Tregs were significantly reduced in the three patient groups compared with controls. Furthermore, in conformity to the downward trend of sFGL2 levels, the HFpEF group had significantly high Tregs frequencies, followed by the HFmrEF group and the HFrEF group (Fig. 3B and C).

Tregs from patients with IHF present compromised suppressive function. Quantitative analysis of the suppressive function of Tregs was performed by co-culturing CD $4^{+} \mathrm{CD} 25^{+}$Tregs and CFSE-labelled $\mathrm{CD} 4^{+} \mathrm{CD} 25^{-}$Tresp cells in different ratios (1:1, 1:2, 1:4 and 1:8) stimulated by anti-CD3/28. As seen in Fig. 4A and B, CD $4^{+} \mathrm{CD} 25^{+}$Tregs from the control and IHF groups were both able to suppress the proliferation of $\mathrm{CD} 4^{+} \mathrm{CD} 25^{-}$Tresp cells from each group. However, compared with controls, Tregs from patients with IHF exhibited impaired ability to suppress proliferation of Tresp cells from the two groups. In addition, the suppressive ability of Tregs was closely related to the ratio of Tregs to Tresp cells.

Over-expressed pro-inflammatory cytokines such as IFN- $\gamma$ and IL-17 participate in immune imbalance in the progress of IHF (9). Thus, the IFN- $\gamma$ and IL-17 levels in the supernatants of the co-culture system were next assessed to investigate whether Tregs were effective to suppress IFN- $\gamma$ and IL-17 production by Tresp cells. Regardless of co-cultured with Tresp cells from controls or patients with IHF, Tregs from patients with
IHF were significantly worse at suppressing IFN- $\gamma$ and IL-17 secretion compared with controls (Fig. 4C and D).

Correlations of serum sFGL2 levels and circulating Tregs frequencies with the indexes of cardiac function. The correlations of serum sFGL2 levels and circulating Tregs frequencies with the indexes of cardiac function in patients with IHF was next analyzed. As shown in Fig. 5A-F, both sFGL2 levels and $\mathrm{CD} 4{ }^{+} \mathrm{CD} 25^{+} \mathrm{Foxp} 3^{+}$Tregs frequencies were positively correlated with LVEF $(r=0.616, \mathrm{P}<0.0001$ and $\mathrm{r}=0.527, \mathrm{P}<0.0001$, respectively), whereas they were negatively correlated with LVEDD $(\mathrm{r}=-0.659, \mathrm{P}<0.0001$ and $\mathrm{r}=-0.536, \mathrm{P}<0.0001$, respectively) and NT-proBNP $(\mathrm{r}=-0.593$, $\mathrm{P}<0.0001$ and $\mathrm{r}=-0.576, \mathrm{P}<0.0001$, respectively) in patients with IHF. Furthermore, it was found that sFGL2 levels were significantly and positively correlated with the frequencies of CD $4^{+} \mathrm{CD} 25^{+}$Foxp $3^{+}$Tregs ( $\mathrm{r}=0.647, \mathrm{P}<0.0001$, Fig. 5G).

Diagnosis efficacy of serum sFGL2 for IHF. As shown in Fig. 6, ROC curve analysis was performed in controls and patients with IHF to assess the diagnosis efficacy of serum sFGL2 in IHF. The area under the curve (AUC) was 0.811 (95\% confidence interval $=0.724-0.897, \mathrm{P}<0.0001)$. The cut-off value for sFGL2 was $13.57 \mathrm{ng} / \mathrm{ml}$, with a sensitivity of $68.0 \%$ and a specificity of $90.6 \%$.

\section{Discussion}

The present study proved for the first time, to the best of the authors' knowledge, that serum sFGL2 levels were markedly decreased in patients with IHF, synchronically accompanied 
A

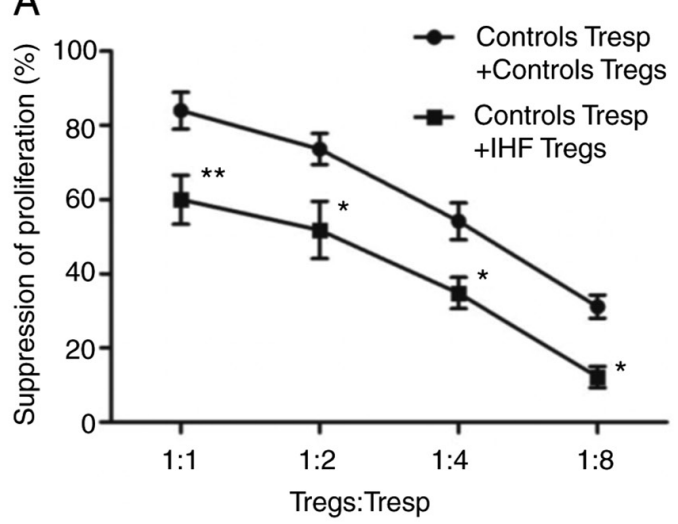

C

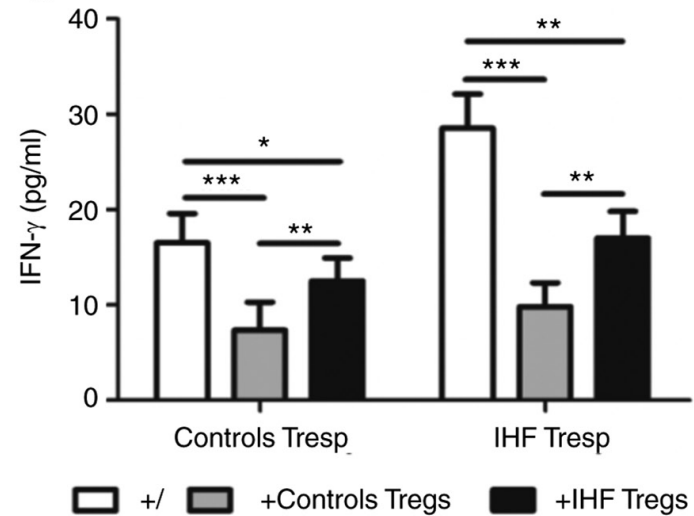

B

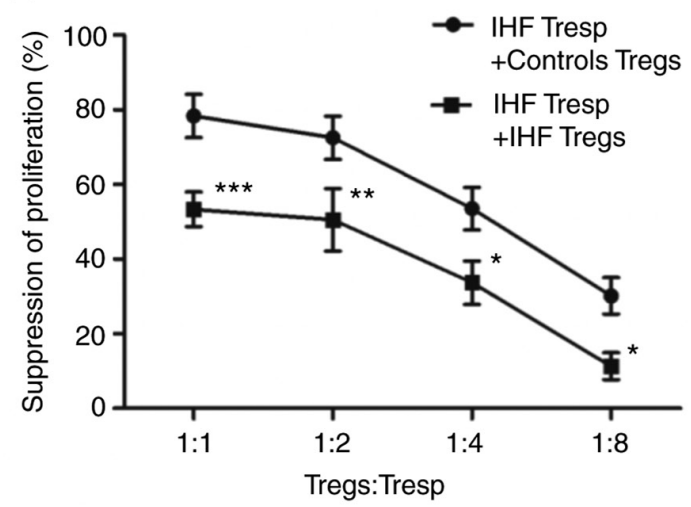

D

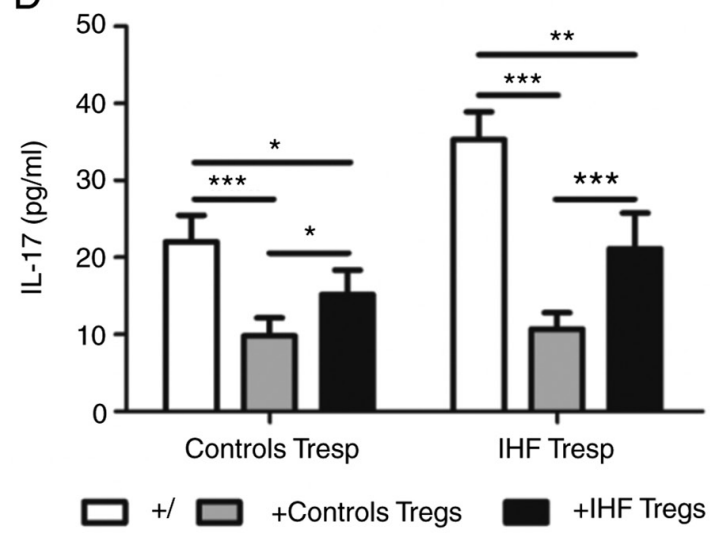

Figure 4. Tregs from patients with IHF present compromised suppressive function. $\mathrm{CD}^{+}{ }^{+} \mathrm{CD} 25^{+}$Tregs and CFSE-labelled CD4 ${ }^{+} \mathrm{CD} 25^{-} \mathrm{T}$ (Tresp) cells were co-cultured at different ratio of 1:1, 1:2, 1:4 and 1:8 for proliferation and suppression assay. Then, four days later, supernatants from the co-cultured $\mathrm{T}$ cells at a 1:1 ratio were assayed for IFN- $\gamma$ and IL-17 by ELISA. (A) Co-cultured with Tresp cells from controls, Tregs from patients with IHF showed reduced suppressive function compared with controls. (B) Co-cultured with Tresp cells from patients with IHF, Tregs from patients with IHF showed reduced suppressive function compared with controls. Tregs from patients with IHF were less effective in suppressing (C) IFN- $\gamma$ and (D) IL-17 secretion compared with controls. ${ }^{*} \mathrm{P}<0.05$, ${ }^{* *} \mathrm{P}<0.01,{ }^{* * *} \mathrm{P}<0.001$. Tregs, regulatory T Cells; IHF, ischemic heart failure; Tresp, responder T cells.

by a pronounced reduction of circulating $\mathrm{CD} 4{ }^{+} \mathrm{CD} 25^{+} \mathrm{Foxp} 3^{+}$ Tregs frequencies and compromised Tregs suppressive function. In patients with IHF, sFGL2 levels and Tregs frequencies were decreased with the deterioration of cardiac function. In addition, a positive correlation was observed between serum sFGL2 levels and LVEF and a significant inverse correlation between serum sFGL2 levels and LVEDD and NT-proBNP. Moreover, sFGL2 levels were significantly and positively correlated with the frequencies of $\mathrm{CD} 4^{+} \mathrm{CD} 25^{+} \mathrm{Foxp} 3^{+}$Tregs.

In this research, all patients enrolled had IHF and had had a long course of the disease. Prior to developing heart failure, some of them had severe coronary stenosis and/or myocardial infarction and received revascularization. Existing myocardial damage and inflammation eventually lead to heart failure (3). By the time the specimens were collected for the present study, the patients had received revascularization long ago. The previous revascularization did not alter or modify the cardiovascular markers, such as LVEF and LVEDD.

With more emerging evidence, imbalance between proand anti-inflammatory responses has been associated with the pathophysiologic process of IHF $(4,5,8)$. Following MI injury, cell debris, reactive oxygen species and proteases are generated immediately and attract inflammatory cells such as neutrophils, macrophages and T cells to the infarcted heart. These immune cells secrete large amounts of proinflammatory cytokines and chemokines and mediate cardiac injury $(8,16)$. $\mathrm{CD} 4^{+} \mathrm{CD} 25^{+} \mathrm{Foxp}^{+}$Treg cells are actively engaged in the control of a variety of physiological and pathological immune responses $(11,26)$. Previous studies have shown that Tregs are increased in murines undergoing experimental MI and recruited to the infarcted myocardium to prevent excessive matrix degradation, limit postinfarction inflammation and ameliorate cardiac remodeling (27-29). As suggested by these studies, the cardioprotective effects of Tregs may be involved in the rebalancing of the immune system and protection against myocardial fibrosis and AVR leading to heart failure. The role can be achieved by suppressing $\mathrm{T}$ cell proliferation and modulating monocyte/macrophage differentiation through direct interaction with target cells and inhibitory cytokines production such as IL-10 and TGF- $\beta(30,31)$. Alternatively, clinical studies have reported that the frequency and function of circulating Tregs were impaired in patients with $\mathrm{CHF}$ and showed a significant inverse correlation with cardiac function $(9,32)$. Consistent with the recent analogous researches, the present study found that circulating Tregs frequencies were decreased in patients with IHF, positively correlated with LVEF and negatively correlated with LVEDD and NT-proBNP. Notably, the suppressive capacity of Tregs on Tresp cells proliferation and pro-inflammatory cytokines secretion was impaired in patients with IHF. This implied that the homeostatic control of Tregs is disordered in 

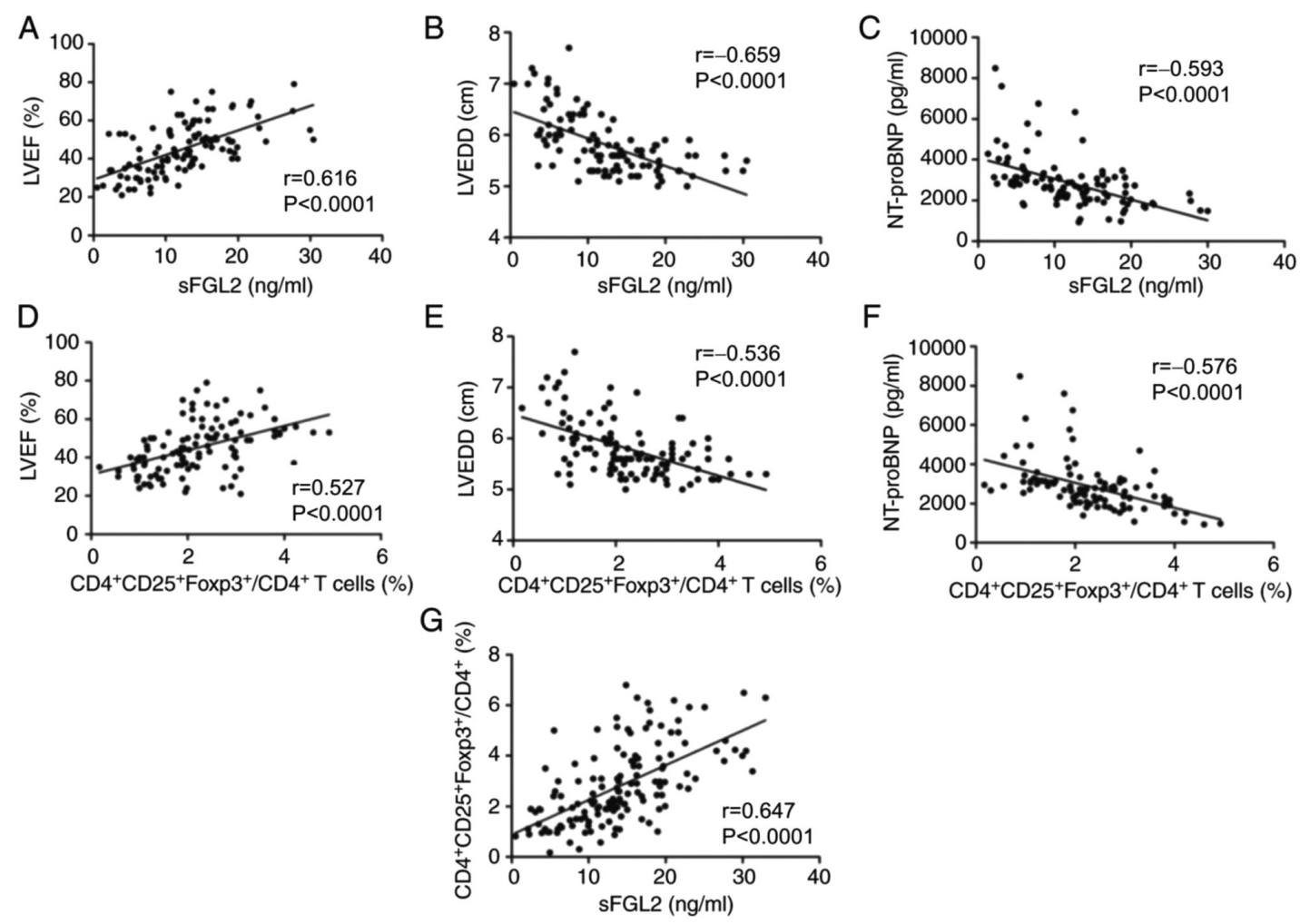

Figure 5. Correlations of serum sFGL2 levels and circulating Tregs frequencies with the indexes of cardiac function. (A-C) Serum sFGL2 levels were positively correlated with (A) LVEF, whereas they were negatively correlated with (B) LVEDD and (C) NT-proBNP. Circulating Tregs frequencies were positively correlated with (D) LVEF, whereas they were negatively correlated with (E) LVEDD and (F) NT-proBNP. (G) sFGL2 levels were positively correlated with Tregs frequencies. sFGL2, soluble fibrinogen-like protein 2; Tregs, regulatory T cells; LVEF, left ventricular ejection fraction; LVEDD, left ventricular end-diastolic dimension; NT-proBNP, N-terminal pro-brain natriuretic peptide.

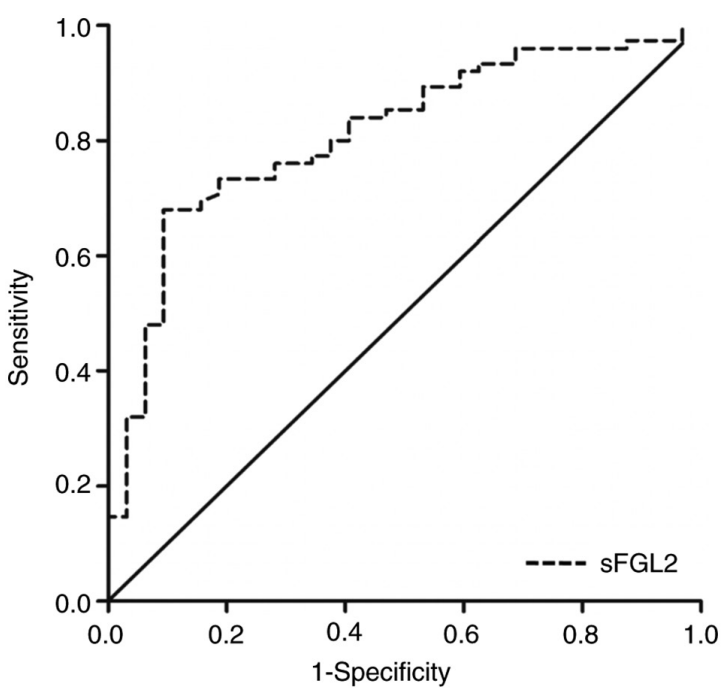

Figure 6. Receiver operating characteristic curve for predicting IHF by serum sFGL2 levels in controls and patients with IHF. The area under the curve and the cut-off value were 0.813 and $13.57 \mathrm{ng} / \mathrm{ml}$, respectively. IHF, ischemic heart failure; sFGL2, soluble fibrinogen-like protein 2.

IHF, while defective Tregs are also correlated with the severity of disease. Given the above, it is possible that impaired Tregs may be associated with the immune imbalance and responsible for the uncontrolled inflammatory response in IHF, which subsequently lead to myocardial damage and deterioration of cardiac function.
sFGL2 is highly expressed in Tregs both at mRNA and protein levels and it contributes to their suppressive activity $(20,23)$. A study on heart transplantation documented that $\mathrm{CD}^{+} \mathrm{T}$ cells in fgl2 transgenic mice exert reduced proliferative activity to alloantigen, anti-CD3 and anti-CD28 stimulation compared with $\mathrm{CD}^{+} \mathrm{T}$ cells in wild-type mice or fgl2 gene knockout mice (33). Simultaneously, the frequencies of splenic Tregs in fgl2 transgenic mice were considerably increased and immunosuppressive activity of Tregs in fgl2 transgenic mice was enhanced in response to ConA stimulation (33). By contrast, sFGL2 deficiency due to gene knockout or antibody blockade can significantly block Treg suppressive function thus leading to immune dysregulation and autoimmune glomerulonephritis (20). However the importance of traditional cytokines released by Tregs such as IL-10 and TGF- $\beta$ remains controversial. Monoclonal antibodies against IL-10 or TGF- $\beta$ fail to inhibit Tregs activity and Tregs obtained from TGF- $\beta$-deficient mice exhibit normal suppressive function $(34,35)$. Moreover, TGF- $\beta$ and IL-10 do not decrease in patients with CHF (10). Therefore, sFGL2 should not only serve as an essential downstream effector of Tregs accounting for suppressive effects other than the traditional anti-inflammatory factors, but it should also increase the frequency and immunosuppressive function of Tregs. Based upon the data of the present study, sFGL2 levels were significantly decreased in the serum of patients with IHF and positively correlated with the frequencies of $\mathrm{CD} 4{ }^{+} \mathrm{CD} 25^{+} \mathrm{Foxp}^{+}$Tregs. This may be explained by impaired 
Tregs that exhibit a deficient capacity to secrete sFGL2 and subsequently by insufficient sFGL2 that downregulates Tregs differentiation and suppressive function, thus forming a vicious circle. sFGL2 has been demonstrated to bind specifically to FcgRIIB and FcgRIII receptors expressed on the surface of macrophages, dendritic cells and lymphocytes to achieve immunosuppression $(22,23)$. Accordingly, the notable reduction of sFGL2 levels, accompanied by decreased Tregs frequencies and compromised suppressive function, might serve a role in the progression of IHF.

In fg12 knockout mice, FGL2 deficiency leads to significant early postnatal lethality due to acute congestive cardiac failure, indicating that FGL2 has an important role in normal cardiac function throughout embryonic and early postnatal development (36). The data of the present study showed that serum levels of sFGL2 were positively correlated with LVEF and were negatively correlated with LVEDD and NT-proBNP, suggesting that sFGL2 was related to the severity of cardiac dilation and dysfunction.

Nevertheless, there are some potential limitations to the present study. It did not calculate the mean fluorescence intensity (MFI) as this study was carried out over a long time span. The samples were collected and tested them in several batches and different batches may present different MFI. In addition, the flow cytometric labeling of Tregs showed high specificity and prominent expression. Thus, the present study only analyzed the proportion of Tregs. If the MFI had been assessed, the present study would have been more rigorous. It also remains uncertain how sFGL2 and Tregs modulate the immune balance in the progression of IHF, resulting in alterations of cardiac function and structure. To solve this problem, the authors of the present study are performing an animal experiment to study the functional roles of sFGL2 and Tregs in wound healing post-MI in a mouse model of permanent left coronary artery ligation. The effects and mechanisms of sFGL2 and Tregs on activation and proliferation of $\mathrm{T}$ cells and differentiation of monocytes and macrophages are being investigated using an experimental MI model of sfgl2 knockout and transgenic mice. Further studies are needed to elucidate whether sFGL2 and Tregs could have clinical applications in the treatment of heart failure.

Taken together, the present study corroborated that serum sFGL 2 levels are decreased in IHF and correlated with cardiac function, suggesting that the reduction of serum sFGL2 levels is associated with IHF progression. As a novel downstream effector of Tregs, sFGL 2 could be a potential indicator for predicting disease severity as well as a therapeutic target for IHF, all of which should be confirmed by further studies.

\section{Acknowledgements}

Not applicable.

\section{Funding}

The present study was supported by the National Natural Science Foundation of China (grant. nos. 81270267 and 81901429).

\section{Availability of data and materials}

The datasets used and/or analyzed during the current study are available from the corresponding author on reasonable request.

\section{Authors' contributions}

YY and SH contributed equally to this study. YY, KL and ZW conceived and designed the study. YY and SH completed the experiments. YY and SH collected blood samples. HL and CF participated in data analysis. YY, KL and ZW wrote the manuscript. All authors confirm the authenticity of all the raw data. All authors read and approved the final version of the manuscript.

\section{Ethics approval and consent to participate}

The present study was approved by the Ethics Committee of Tongji Medical College, Huazhong University of Science and Technology (approval. no. IORG0003571) and written informed consent was obtained from each participant. This investigation conformed to the Declaration of Helsinki principles.

\section{Patient consent for publication}

Not applicable.

\section{Competing interests}

The authors declare that they have no competing interests.

\section{References}

1. Gerber Y, Weston SA, Enriquez-Sarano M, Berardi C, Chamberlain AM, Manemann SM, Jiang R, Dunlay SM and Roger VL: Mortality associated with heart failure after myocardial infarction: A contemporary community perspective. Circ Heart Fail 9: e002460, 2016.

2. Barsheshet A, Moss AJ, Eldar M, Huang DT, Hall WJ, Klein HU, McNitt S, Steinberg JS, Wilber DJ, Zareba W and Goldenberg I: Time-Dependent benefit of preventive cardiac resynchronization therapy after myocardial infarction. Eur Heart J 32: 1614-1621, 2011.

3. Briceno N, Schuster A, Lumley M and Perera D: Ischaemic cardiomyopathy: Pathophysiology, assessment and the role of revascularisation. Heart 102: 397-406, 2016.

4. Tang TT, Zhu YC, Dong NG, Zhang S, Cai J, Zhang LX, Han Y, Xia N, Nie SF, Zhang M, et al: Pathologic T-cell response in ischaemic failing hearts elucidated by T-cell receptor sequencing and phenotypic characterization. Eur Heart J 40: 3924-3933, 2019.

5. Swirski FK and Nahrendorf M: Leukocyte behavior in atherosclerosis, myocardial infarction, and heart failure. Science 339: 161-166, 2013

6. Wang Z, Huang S, Sheng Y, Peng X, Liu H, Jin N, Cai J, Shu Y, Li T, Li P, et al: Topiramate modulates post-infarction inflammation primarily by targeting monocytes or macrophages. Cardiovasc Res 113: 475-487, 2017.

7. Yan X, Anzai A, Katsumata Y, Matsuhashi T, Ito K, Endo J, Yamamoto T, Takeshima A, Shinmura K, Shen W, et al: Temporal dynamics of cardiac immune cell accumulation following acute myocardial infarction. J Mol Cell Cardiol 62: 24-35, 2013.

8. Prabhu SD and Frangogiannis NG: The biological basis for cardiac repair after myocardial infarction: From inflammation to fibrosis. Circ Res 119: 91-112, 2016. 
9. Tang TT, Ding YJ, Liao YH, Yu X, Xiao H, Xie JJ, Yuan J, Zhou ZH, Liao MY, Yao R, et al: Defective circulating CD4CD25+Foxp3+CD127(low) regulatory T-cells in patients with chronic heart failure. Cell Physiol Biochem 25: 451-458, 2010.

10. Tang TT, Zhu ZF, Wang J, Zhang WC, Tu X, Xiao H, Du XL, Xia JH, Dong NG, Su W, et al: Impaired thymic export and apoptosis contribute to regulatory T-cell defects in patients with chronic heart failure. PLoS One 6: e24272, 2011

11. Dominguez-Villar M and Hafler DA: Regulatory T cells in autoimmune disease. Nat Immunol 19: 665-673, 2018.

12. Zhu ZF, Tang TT, Dong WY, Li YY, Xia N, Zhang WC, Zhou SF, Yuan J, Liao MY, Li JJ, et al: Defective circulating CD4+LAP+ regulatory $\mathrm{T}$ cells in patients with dilated cardiomyopathy. J Leukoc Biol 97: 797-805, 2015.

13. Chen XH, Ruan CC, Ge Q, Ma Y, Xu JZ, Zhang ZB, Lin JR, Chen DR, Zhu DL and Gao PJ: Deficiency of complement C3a and $\mathrm{C} 5 \mathrm{a}$ receptors prevents angiotensin II-induced hypertension via regulatory T cells. Circ Res 122: 970-983, 2018.

14. Meiler S, Smeets E, Winkels H, Shami A, Pascutti MF, Nolte MA, Beckers L, Weber C, Gerdes N and Lutgens E: Constitutive GITR activation reduces atherosclerosis by promoting regulatory CD4+ T-cell responses-brief report. Arterioscler Thromb Vasc Biol 36: 1748-1752, 2016

15. Flego D, Severino A, Trotta F, Previtero M, Ucci S, Zara C Massaro G, Pedicino D, Biasucci LM, Liuzzo G and Crea F: Increased PTPN22 expression and defective CREB activation impair regulatory $\mathrm{T}$-cell differentiation in non-ST-segment elevation acute coronary syndromes. J Am Coll Cardiol 65: 1175-1186, 2015.

16. Tang TT, Yuan J, Zhu ZF, Zhang WC, Xiao H, Xia N, Yan XX, Nie SF, Liu J, Zhou SF, et al: Regulatory T cells ameliorate cardiac remodeling after myocardial infarction. Basic Res Cardiol 107: 232, 2012

17. Meng X, Yang J, Dong M, Zhang K, Tu E, Gao Q, Chen W, Zhang $\mathrm{C}$ and Zhang Y: Regulatory T cells in cardiovascular diseases. Nat Rev Cardiol 13: 167-179, 2016.

18. Long R, You Y, Li W, Jin N, Huang S, Li T, Liu K and Wang Z: Sodium tanshinone IIA sulfonate ameliorates experimental coronary no-reflow phenomenon through down-regulation of FGL2 . Life Sci 142: 8-18, 2015

19. Li WZ, Wang J, Long R, Su GH, Bukhory DK, Dai J, Jin N, Huang SY, Jia P, Li T, et al: Novel antibody against a glutamic acid-rich human fibrinogen-like protein 2-derived peptide near Ser91 inhibits hfgl2 prothrombinase activity. PLoS One 9: e94551, 2014

20. Shalev I, Liu H, Koscik C, Bartczak A, Javadi M, Wong KM, Maknojia A, He W, Liu MF, Diao J, et al: Targeted deletion of fgl2 leads to impaired regulatory $\mathrm{T}$ cell activity and development of autoimmune glomerulonephritis. J Immunol 180: 249-260, 2008.

21. Zhao Z, Yang C, Wang L, Li L, Zhao T, Hu L, Rong R, Xu M and Zhu T: The regulatory $\mathrm{T}$ cell effector soluble fibrinogen-like protein 2 induces tubular epithelial cell apoptosis in renal transplantation. Exp Biol Med (Maywood) 239: 193-201, 2014

22. Foerster K, Helmy A, Zhu Y, Khattar R, Adeyi OA, Wong KM Shalev I, Clark DA, Wong PY, Heathcote EJ, et al: The nove immunoregulatory molecule FGL2: A potential biomarker for severity of chronic hepatitis C virus infection. J Hepatol 53: 608-615, 2010

23. Chan CW, Kay LS, Khadaroo RG, Chan MWC, Lakatoo S, Young KJ, Zhang L, Gorczynski RM, Cattral M, Rotstein O and Levy GA: Soluble fibrinogen-like protein 2/fibroleukin exhibits immunosuppressive properties: Suppressing T cell proliferation and inhibiting maturation of bone marrow-derived dendritic cells. J Immunol 170: 4036-4044, 2003.
24. Liu K, Li T, Huang S, Long R, You Y, Liu J and Wang Z: The reduced soluble fibrinogen-like protein 2 and regulatory $T$ cells in acute coronary syndrome. Exp Biol Med (Maywood) 241: 421-425, 2016

25. Ponikowski P, Voors AA, Anker SD, Bueno H, Cleland John GF, Coats Andrew JS, Falk V, González-Juanatey JR, Harjola VP, Jankowska EA, et al: 2016 ESC Guidelines for the diagnosis and treatment of acute and chronic heart failure: The task force for the diagnosis and treatment of acute and chronic heart failure of the European Society of Cardiology (ESC). Developed with the special contribution of the Heart Failure Association (HFA) of the ESC. Eur J Heart Fail 37: 2129-2200, 2016.

26. Kasper IR, Apostolidis SA, Sharabi A and Tsokos GC: Empowering regulatory $\mathrm{T}$ cells in autoimmunity. Trends $\mathrm{Mol}$ Med 22: 784-797, 2016.

27. Sharir R, Semo J, Shimoni S, Ben-Mordechai T, Landa-Rouben N, Maysel-Auslender S, Shaish A, Entin-Meer M, Keren G and George J: Experimental myocardial infarction induces altered regulatory $\mathrm{T}$ cell hemostasis, and adoptive transfer attenuates subsequent remodeling. PLoS One 9: e113653, 2014.

28. Saxena A, Dobaczewski M, Rai V, Haque Z, Chen W, Li N and Frangogiannis NG: Regulatory $\mathrm{T}$ cells are recruited in the infarcted mouse myocardium and may modulate fibroblast phenotype and function. Am J Physiol Heart Circ Physiol 307: H1233-H1242, 2014.

29. Ramjee V, Li D, Manderfield LJ, Liu F, Engleka KA Aghajanian H, Rodell CB, Lu W, Ho V, Wang T, et al: Epicardial YAP/TAZ orchestrate an immunosuppressive response following myocardial infarction. J Clin Invest 127: 899-911, 2017.

30. Lu L, Barbi J and Pan F: The regulation of immune tolerance by FOXP3. Nat Rev Immunol 17: 703-717, 2017.

31. Weirather J, Hofmann UD, Beyersdorf N, Ramos GC, Vogel B, Frey A, Ertl G, Kerkau T and Frantz S: Foxp3+ CD4+ T cells improve healing after myocardial infarction by modulating monocyte/macrophage differentiation. Circ Res 115: 55-67, 2014

32. Li N, Bian H, Zhang J, Li X, Ji X and Zhang Y: The Th17/treg imbalance exists in patients with heart failure with normal ejection fraction and heart failure with reduced ejection fraction. Clin Chim Acta 411: 1963-1968, 2010.

33. Bartczak A, Chruscinski A, Mendicino M, Liu H, Zhang J, He W, Amir AZ, Nguyen A, Khattar R, Sadozai H, et al: Overexpression of fibrinogen-like protein 2 promotes tolerance in a fully mismatched murine model of heart transplantation. Am J Transplant 16: 1739-1750, 2016.

34. Tang Q, Boden EK, Henriksen KJ, Bour-Jordan H, Bi M and Bluestone JA: Distinct roles of CTLA-4 and TGF-beta in CD4+CD25+ regulatory T cell function. Eur J Immunol 34: 2996-3005, 2004.

35. Miyara $M$ and Sakaguchi S: Natural regulatory $T$ cells: Mechanisms of suppression. Trends Mol Med 13: 108-116, 2007.

36. Mu J, Qu D, Bartczak A, Phillips MJ, Manuel J, He W, Koscik C, Mendicino M, Zhang L, Clark DA, et al: Fgl2 deficiency causes neonatal death and cardiac dysfunction during embryonic and postnatal development in mice. Physiol Genomics 31: 53-62, 2007.

This work is licensed under a Creative Commons Attribution-NonCommercial-NoDerivatives 4.0 International (CC BY-NC-ND 4.0) License. 